\title{
Role of physical activity in mortality prediction in elderly hospice patients: the methodological issues
}

\author{
Kamyar Mansori ${ }^{1,2}$, Fatemeh Khosravi Shadmani ${ }^{3}$, Shiva Mansouri Hanis ${ }^{4}$, Salman Khazaei ${ }^{5, *}$ \\ 'Social Development \& Health Promotion Research Center, Gonabad University of Medical Sciences, Gonabad, Iran \\ ${ }^{2}$ Department of Epidemiology, School of Public Health, Iran University of Medical Science, Tehran, Iran \\ ${ }^{3}$ Modeling in Health Research Center, Institute for Futures Studies in Health, Kerman University of Medical Sciences, Kerman, Iran \\ ${ }^{4}$ Department of Epidemiology, School of Public Health, Shahid Beheshti University of Medical Sciences, Tehran, Iran \\ ${ }^{5}$ Department of Epidemiology, School of Public Health, Hamadan University of Medical Sciences, Hamadan, Iran
}

\section{To the editor:}

We were interested to read the paper by Hye-Sang Park with entitled "Role of physical activity in mortality prediction in elderly hospice patients "which was published in Journal of Exercise Rebabilitation in April 2017. The aim of this study was to investigate the mortality predictors and indicators of life extension among elderly patients in a hospice service center. The results revealed that the time of death negatively correlated with activities of daily living (ADL) score $(r=-0.273, P=0.006)$. However, the Korean-Mini Mental State Examination \& Global Deterioration Scale, and the values of blood component such as albumin, gamma-glutamic transpeptidase, blood urea nitrogen, and creatinine were not correlated with the time of death. Consequently, the ADL score might be an important predictor of mortality and life extension in elderly patients. Finally, this study concludes that in order to improve ADL score, physical exercise and overall fitness may be potential nonpharmacologic methods useful in preventing mortality in elderly people (Park et al., 2017). However, regardless of the results of the study that are interesting, some methodological issues should be considered relating to this cross-sectional study.

First, for prediction studies, we need data from two different cohorts or at least from one cohort divided into two to first to develop a prediction model and subsequently validate it. Misleading results are generally the main outcome of research that fails to validate its prediction models (Rothman et al., 2008; Sani et al., 2016;
Sabour, 2017). And also, without the temporality assumption (the dependent variable must occur after the independent variable) there is no way of determining whether a factor is a risk factor, is predictive/causal, or is a consequence of the outcome (Hanis et al., 2017; Mansori et al., 2017). Therefore, longitudinal studies are essential for developing assumptions to be used in clinical prediction models, whereas in this study, a cross-sectional study was used to identify the independent predictors for mortality prediction in elderly hospice patients. Therefore, it is essential to interpret the results of this study in light of the above explanation.

\section{CONFLICT OF INTEREST}

No potential conflict of interest relevant to this article was reported.

\section{REFERENCES}

Hanis SM, Shadmani FK, Mansori K. Letter to editor: The waist circumference-adjusted associations between hyperuricemia and other lifestyle-related diseases: methodological issues in cross-sectional study. Diabetol Metab Syndr 2017;9:23.

Mansori K, Mansouri Hanis S, Shadmani FK. Postpartum modern contraceptive use in northern Ethiopia: prevalence and associated factors methodological issues in this cross-sectional study. Epidemiol Health

\footnotetext{
${ }^{*}$ Corresponding author: Salman Khazaei

(D) https://orcid.org/0000-0001-5918-2310

Department of Epidemiology, School of Public Health, Hamadan University of

Medical Sciences, Hamadan, Iran

Tel: +98-9212848625, Fax: +98-9212848625, E-mail: salman.khazaei61@gmail.com

Received: June 19, 2017 / Accepted: August 7, 2017
}

This is an Open Access article distributed under the terms of the Creative Commons Attribution Non-Commercial License (http://creativecommons.org/licenses/by-nc/4.0/) which permits unrestricted non-commercial use, distribution, and reproduction in any medium, provided the original work is properly cited. 
2017;39:e2017019.

Park HS, Lee KT, Kim TW. Role of physical activity in mortality prediction in elderly hospice patients. J Exerc Rehabil 2017;13:250-254.

Rothman KJ, Greenland S, Lash TL. Modern epidemiology. Philadelphia (PA): Lippincott Williams \& Wilkins; 2008.

Sabour S. Prediction of rehabilitation needs after treatment of cervical cancer: a methodological mistake. Support Care Cancer 2017;25:2041.

Sani M, Ayubi E, Mansori K, Khazaei S. Predictive ability of HAS-BLED, HEMORR2HAGES, and ATRIA bleeding risk scores in patients with atrial fibrillation: Methodological issues of prediction models. Int J Cardiol 2016;222:949. 